\title{
GESTÃO DAS INOVAÇÕES INCREMENTAIS, O CASO OMEPRAZOLA
}

\author{
Alexandre Lopes Lourenço* e Luiz Marcelo Lira \\ Instituto Nacional da Propriedade Industrial, Rua Mayrink Veiga, 9, 20090-910 Rio de Janeiro - RJ, Brasil \\ Denise Pires de Carvalho \\ Instituto de Biofísica Carlos Chagas Filho, Centro de Ciências da Saúde, Bl. G, Universidade Federal do Rio de Janeiro, Ilha do \\ Fundão, 21949-900 Rio de Janeiro - RJ, Brasil \\ Paulo de Assis Melo e Tereza Sollero Cláudio-da-Silva \\ Instituto de Ciências Biomédicas, Centro de Ciências da Saúde, Bl. K, Universidade Federal do Rio de Janeiro, Ilha do Fundão, \\ 21941-590 Rio de Janeiro - RJ, Brasil \\ Recebido em 15/12/08; aceito em 14/10/09; publicado na web em 25/3/10
}

\begin{abstract}
MANAGEMENT OF INCREMENTAL INNOVATIONS, THE CASE OMEPRAZOLE. Our report shows the strategies adopted by pharmaceutical industry in the development of incremental innovations, through the development carried out after the launch of omeprazole. Some improvements are really necessary and others are used only as a market strategy and evergreening.
\end{abstract}

Keywords: incremetal innovation; omeprazole; patent.

\section{INTRODUÇÃO}

A essência do sistema patentário é estimular a inventividade, encorajar o progresso técnico e possibilitar a disseminação das inovações, justificando, desta forma, a restrição ao livre acesso às tecnologias patenteadas (monopólio temporário) como forma de retribuição financeira ao investimento necessário para o seu desenvolvimento e como fomento às novas invenções. ${ }^{1} \mathrm{O}$ incentivo ao desenvolvimento de diversas novas tecnologias está vinculado à obtenção do direito de exclusividade em sua comercialização. ${ }^{2,3} \mathrm{Na}$ área farmacêutica o sistema permite diversos tipos de proteção, podendo uma mesma molécula obter outras proteções por patentes através de melhorias desenvolvidas por pesquisas posteriores - "inovações incrementais". ${ }^{4}$ A possibilidade de inovar partindo de um produto já conhecido é frequentemente explorada pela indústria farmacêutica. ${ }^{5}$ Tais inovações, também denominadas "inovações sequenciais", não representam um salto tecnológico muito grande, e demandam um nível de investimento muito menor que as inovações chamadas de "radicais". Entretanto, desempenham papel importante, servindo para equilibrar o mercado, uma vez que permitem o surgimento da concorrência antes do término de vigência da patente, fornecendo, por exemplo, opções de escolha entre fármacos estruturalmente similares me too ou me better. ${ }^{4}$ Também podem promover maior comodidade ao tratamento através de novas formas farmacêuticas ou promover melhorias farmacotécnicas com alteração da estabilidade, solubilidade, biodisponibilidade, como no caso do desenvolvimento de sais, hidratos, cocristais e suas diferentes formas polimórficas. ${ }^{6}$ Outro ponto importante é a melhoria do perfil de segurança que pode ser obtida através de processos de síntese estereosseletiva ou de técnicas de purificação que permitam o uso na fabricação do medicamento da forma ativa enantiomericamente pura (eutômero), ${ }^{7}$ com a diminuição dos efeitos adversos provocados pela outra forma enantiomérica (distômero). ${ }^{7}$ Melhorias no processo de obtenção da molécula também são muito comuns, uma vez que a primeira patente depositada normalmente traz as etapas de produção em pequena escala, sendo necessárias diversas adaptações para a produção em escala industrial (scale-up) e mesmo os processos já estabelecidos em escala industrial podem sofrer modificações que melhorem o seu rendimento,

\footnotetext{
*e-mail: allopes@inpi.gov.br
}

reduzam os custos de produção, reduzam a geração de contaminantes, entre outros problemas existentes nos primeiros processos.

Apesar dos diversos benefícios que as inovações incrementais podem trazer, alguns autores têm questionado a proteção por patentes desse tipo de tecnologia, uma vez que poderia ser utilizada, pelo detentor da primeira patente, como estratégia para a extensão da vigência da mesma, prática conhecida como evergreening. Desta forma, servindo como desincentivo à inovação radical, com consequente diminuição do investimento na produção de novas entidades moleculares.?

Não é nosso objetivo promover uma discussão de mérito ou dos impactos dessas patentes no incentivo às inovações, mas sim apresentar, através de exemplos, as estratégias adotadas pela indústria farmacêutica no desenvolvimento das inovações incrementais e na gestão do patrimônio tecnológico.

\section{ESTRATÉGIA DE AQUISIÇÃO DE INFORMAÇÕES}

Como forma de exemplificar a estratégia de desenvolvimento e gestão da indústria farmacêutica, escolhemos levantar os caminhos seguidos na gestão de desenvolvimento adotada para uma molécula. Para escolha dessa molécula partimos dos três medicamentos com maior rentabilidade no momento (Tabela 1) e dentro destes o que tem maior número de pedidos de patente. Na escolha dos documentos patentários utilizados não foram diferenciados os que tiveram a patente concedida ou não, uma vez que não é a nossa finalidade discutir o mérito dessas melhorias.

Tabela 1. Fármacos mais vendidos no mercado (em valor) no ano de 2008

\begin{tabular}{lcc}
\hline Fármaco / Medicamento & Venda anual \$ Bilhões & $\%$ mercado \\
\hline Atorvastatina $\left(\right.$ Lipitor $\left.^{\circledR}\right)$ & 13,6 & 2,0 \\
Clopidogrel $\quad\left(\right.$ Plavix $\left.^{\circledR}\right)$ & 7,9 & 1,1 \\
Esomeprazola $\left(\right.$ Nexium $\left.^{\circledR}\right)$ & 7,4 & \\
\hline
\end{tabular}

Fonte: INTERFARMA

Como forma de rastrear as patentes ligadas a estas moléculas realizamos consulta ao sítio do Food and Drug Administation (FDA), na página do eletronic orange book, onde está disponível a informação 
da patente ligada a cada fármaco aprovado para comercialização no mercado americano. Com as informações obtidas na primeira patente concedida para cada fármaco, realizamos busca no sítio do United States Patent and Trademark Office (USPTO) ${ }^{7}$ e do European Patent Office (EPO). ${ }^{8}$ Os documentos encontrados foram analisados quanto ao escopo das modificações realizadas no processo de obtenção da molécula, na nova forma farmacêutica, nos análogos estruturais ou na produção de sais, hidratos, cocristais ou de suas formas polimórficas, além de avaliar a existência de outros usos.

\section{Descrição dos resultados}

Na busca ao sítio do FDA foram obtidas 11 patentes para a atorvastatina, 4 para o clopidogrel e 40 para a omeprazola (primeiro depósito da esomeprazola). Desta forma, a omeprazola foi a molécula utilizada como, nosso modelo de estudo (Figuras 1, 2 e 3 ).

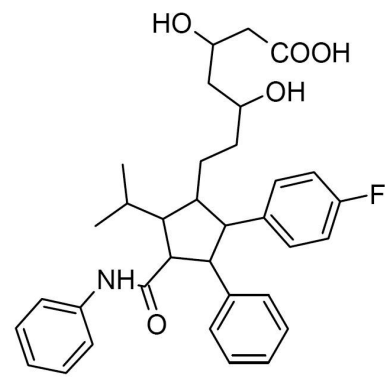

Figura 1. Atorvastatina<smiles>COC(=O)C(c1ccccc1Cl)N1CCc2sccc2C1</smiles>

Figura 2. Clopidogrel<smiles>COc1ccc2c(c1)CC(S(=O)Oc1ncc(C)c(OC)c1C)C2</smiles>

Figura 3. Estrutura da omeprazola

A omeprazola é uma molécula inibidora da secreção ácida gástrica, que foi desenvolvida pela empresa sueca Aktiebolaget Kassle, com propriedade transferida posteriormente para Astra Aktiebolaget, sendo hoje da Astra Zeneca. Este composto foi citado pela primeira vez no documento patentário, depositado no escritório sueco, SE7402101 de $18 / 2 / 1974,{ }^{11}$ sendo descrito dentro da fórmula do tipo markush (Figura 4 e Tabela 2).

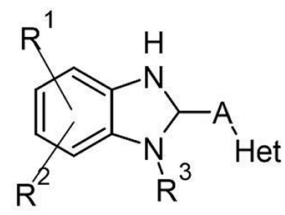

Figura 4. Fórmula do tipo markush do primeiro depósito da omeprazola, SE7402101
Tabela 2. Possíveis substituintes da fórmula tipo markush depositada que continha a molécula da omeprazola

R1 e R2 - podem ser: H, alquila, halogênio, N, carboxila, hidroxialquila, carboxialquila, hidrolila, alcooxila, hidroxialquila, alcanoila, em qualquer posição do anel.

R3 - pode ser: H, alcanoila e carboxila.A pode ser: $\mathrm{SCH} 2-$ e $\mathrm{SCH}(\mathrm{CH} 3)-$

Fonte: SE7402101 ${ }^{8}$

No primeiro documento é reivindicado um método de inibição da secreção ácida em mamíferos, com o uso dos referidos compostos, seus sais terapeuticamente aceitáveis e uma composição farmacêutica para inibir a secreção ácida, o processo de obtenção destes compostos e os compostos, não sendo exemplificado nenhum dos sais farmaceuticamente úteis.

A molécula omeprazola mostrou-se muito pouco solúvel em água e extremamente lábil em pH ácido, com uma razão de dissolução que se apresentava como um fator limitante para absorção, ocorrendo também degradação pré-absorção, devido ao $\mathrm{pH}$ estomacal. Estas dificuldades levaram ao desenvolvimento de uma forma farmacêutica para uso oral, ${ }^{12}$ consistindo de um núcleo contendo a omeprazola com uma substância alcalina, uma camada de separação solúvel em água ou polímeros solúveis em água, opcionalmente recoberto por uma camada de liberação entérica.

O desenvolvimento não parou, havendo um intenso trabalho de pesquisa que propiciou uma série de melhorias na forma farmacêutica, o aperfeiçoamento dos processos de produção e a alteração das características da forma cristalina, com mudança na estabilidade e solubilidade. Estas alterações incrementais são descritas de forma resumida abaixo.

Como não haviam sido especificamente citados os sais na primeira patente, foi depositado um pedido de patente contendo novos sais de adição da omeprazola $\left(\mathrm{Li}^{+}, \mathrm{Na}^{+}, \mathrm{K}^{+}, \mathrm{Mg} 2^{+}, \mathrm{Ca} 2+, \mathrm{Ti}^{+}\right),{ }^{13}$ bem como o seu processo de preparação. São reivindicadas também as composições contendo esses sais, que trazem como melhoria maior estabilidade quando comparada às formulações preparadas com o composto original. Proporcionando menor degradação durante os processos de produção das formas farmacêuticas e permitindo aumentar o tempo de prateleira.

Na época do depósito da patente US5690960, ${ }^{14}$ já se conheciam o composto e os seus diferentes sais, incluindo o sal de magnésio de omeprazola, além de diversas formas farmacêuticas para administração oral, sendo agora apresentada uma pequena melhoria no processo de produção em larga escala dos sais de adição, permitindo a obtenção de um produto mais cristalino, com maior grau de pureza, menor quantidade de água e baixo teor dos outros solventes utilizados no processo.

Os cristais obtidos por este processo propiciaram também maior facilidade de uso na produção galênica, devido à maior estabilidade. No tocante às reivindicações, neste pedido também são requeridas uma nova forma farmacêutica contendo o sal de magnésio de omeprazola, o sal de adição comercialmente utilizado nas formas farmacêuticas posteriores a este depósito.

Outros processos de produção do sal de magnésio foram desenvolvidos, buscando sempre melhor rendimento da forma cristalina, com aumento da estabilidade e consequente melhoria no processo de produção da forma farmacêutica. Outro exemplo está contido na patente US5900424 onde o processo além de melhorar o rendimento, ${ }^{15}$ também fornece cristais de tamanho padronizados entre 20 e $30 \mu \mathrm{m}$.

A forma racêmica da omeprazola (Losec ${ }^{\circledast}, 1988$; Prilosec $^{\circledast}, 1990$ ) foi comercializada, tornando-se um sucesso de venda, blockbuster. Entretanto, uma vez que os estudos de farmacocinética demonstraram que a omeprazola era metabolizada principalmente pelas enzimas do complexo citocromo P450, CYP2C19 e CYP3A4, e que, no entanto, o 
metabolismo ocorria de forma diferenciada para as duas formas - (S) e (R). Com a finalidade de melhorar a sua disponibilidade foi então utilizada a forma (S) do omeprazola $\left(\right.$ Nexium $\left.^{\circledR}\right)$, que apresentava metabolismo mais lento que a forma (R), levando a formulações farmacêuticas com um perfil farmacocinético diferente das anteriormente comercializadas. ${ }^{16}$

Essa estratégia desencadeou uma série de novos depósitos de patentes contendo processos de produção com obtenção do composto na forma enantiomérica com maior grau de pureza. Foram utilizados vários recursos, como a geração de intermediários dos enantiômeros ou pela oxidação do sulfeto pró-quiral, por exemplo, usando um complexo de titânio quiral e um agente de oxidação. ${ }^{17,18}$

A obtenção da forma (S), somada à melhora da biodisponibilidade, permitiu o pedido de método terapêutico utilizando o novo sal, uma vez que o mesmo fornecia um efeito terapêutico melhor quando comparado ao anterior. ${ }^{18}$ Outras modificações realizadas no processo de produção, como a inclusão de uma etapa de recristalização em solução aquosa de $\mathrm{NaOH}$ com pH 9,0, dispensando o uso de solventes orgânicos não alcoólicos, permitiram a obtenção de cristais mais puros como, por exemplo, cristais contendo uma quantidade indetectável por HPLC de solventes orgânicos. ${ }^{19}$

Voltando aos aperfeiçoamentos galênicos, um exemplo são as formas de administração oral e a produção de peletes com um núcleo contendo o ingrediente ativo somado a um composto alcalino, e um excipiente farmaceuticamente aceitável, um agregante, ou peletes produzidos com um núcleo sem ativo, sendo o ativo adicionado em uma segunda camada. Posteriormente a estas etapas, o núcleo era recoberto por uma camada de separação e, posteriormente, por uma fina camada de revestimento entérico, um polímero. Por exemplo, a hidroxipropilcelulose (HPC) ou hidroxipropiletilcelulose (HPMC), onde a variação nas características dos polímeros ou nas camadas poderia alterar a disponibilidade do fármaco. ${ }^{20}$ Também foram descritas formas farmacêuticas em comprimidos, nas quais ocorre a introdução de uma camada entérica polimérica com maior resistência mecânica permitindo a compressão dos peletes, podendo estes comprimidos conter opcionalmente uma camada de proteção gástrica. ${ }^{21,22}$

Partindo das técnicas disponíveis para fabricação de peletes, outra adaptação farmacotécnica foi a utilização de diferentes camadas contendo o fármaco intercaladas por camadas de retardo, com uma última camada de liberação entérica. Isso permite uma liberação gradual do fármaco (Figura 5) ou, ainda, com um atraso maior entre as camadas de liberação permitindo obter uma forma de liberação pulsátil com dois ou mais picos do fármaco em intervalos determinados.

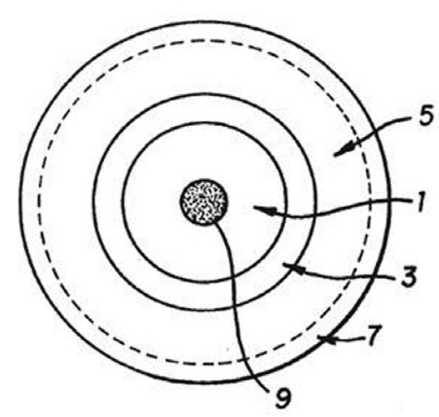

Figura 5. Forma de liberação controlada com diferentes camadas, US5413777

A exploração dos diferentes graus de hidratação possíveis para a molécula dos sais de omeprazola foi outra estratégia utilizada pela indústria para pequenas melhorias na estabilidade da molécula como, por exemplo, o processo apresentado na patente US6369085, ${ }^{18}$ onde se obtém uma estabilidade melhorada dos cristais formados na molécula tri-hidratada. Na mesma linha, o estudo das diferentes formas polimórficas dos cristais de omeprazola levou ao desenvolvimento de processos para obtenção de cristais com alto grau de pureza da forma polimórfica, que apresentava alguma vantagem frente às outras. Um exemplo desses processos é apresentado na US2007/0072913, ${ }^{20}$ onde a forma A da omeprazola é termodinamicamente mais estável que a forma $\mathrm{B}$.

O sucesso de mercado da omeprazola levou a uma busca por moléculas análogas, me too, pelos concorrentes como, por exemplo, a lansoprazola, a pantoprazola e a rabeprazola (Figura 6)..$^{24,25}$<smiles>COc1ccnc(OS(=O)c2nc3ccc(OC(F)F)cc3[nH]2)c1OC</smiles>

A

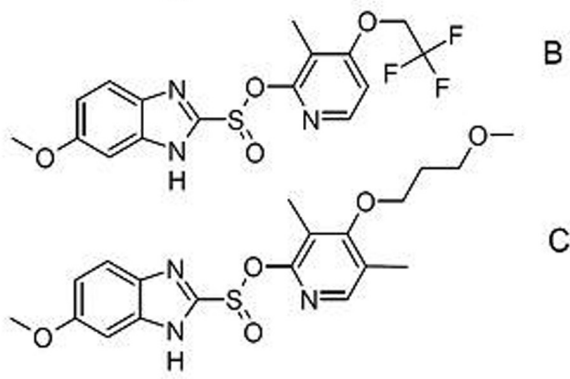

Figura 6. Estruturas dos principais análogos estruturais da omeprazola: (A) pantoprazola, (B) lansoprazola e $(C)$ rabeprazola

Outro aspecto que pode ser observado é a atividade da molécula em outros alvos farmacológicos propiciando, desta maneira, a produção de medicamentos com outras indicações terapêuticas, por exemplo, a US5714505 que descreve o uso da omeprazola para o tratamento da psoríase ou a US5093342,23, ${ }^{27}$ que descreve a sua atividade antimicrobiana.

\section{DISCUSSÃO}

O nosso objetivo foi demonstrar a estratégia normalmente adotada pelos laboratórios farmacêuticos, na gestão da inovação após a introdução no mercado de uma nova entidade molecular. Uma vez que a entrada dessa nova molécula promove o início de uma série de outras pesquisas, cujos produtos são chamados de "inovações incrementais ou sequenciais".

Estas pesquisas têm como foco o aperfeiçoamento da molécula inventada, a adaptação para as diferentes formas de administração ou melhoria dos processos de produção. Ademais, uma molécula para um novo alvo farmacológico e com grande sucesso de mercado gera, por parte dos concorrentes, pesquisas na busca de análogos moleculares, na tentativa de também entrar no mercado.

A omeprazola surge como alternativa ao tratamento das doenças relacionadas com o excesso de acidez gástrica (doenças ácidopépticas). Seu desenvolvimento surgiu da criação de uma linha de pesquisa direcionada aos inibidores da secreção gástrica, iniciada em 1960 pela Hässle. Entretanto, as primeiras pesquisas, que utilizavam ratos como modelo experimental, resultaram em compostos que não reproduziram a sua atividade em humanos, levando à interrupção do programa. ${ }^{28} \mathrm{Em} \mathrm{1972,} \mathrm{o} \mathrm{projeto} \mathrm{foi} \mathrm{retomado} \mathrm{usando} \mathrm{agora} \mathrm{o} \mathrm{modelo}$ de fistula gástrica em cachorros anestesiados. As pesquisas publicadas sobre o assunto continham um composto desenvolvido pela Servier (CMN 131), que apresentava um grupo tioamida e era responsável por uma severa toxicidade aguda. ${ }^{28} \mathrm{~A}$ estratégia adotada foi incorporar este grupo ou internamente ou entre um sistema de anéis heterocíclicos. 
Em 1973, o primeiro hit foi desenvolvido (benzimidazol H 124/26) e se mostrou um potente antissecretor, sem apresentar toxicidade aguda. ${ }^{11,28} \mathrm{O}$ interessante é que este composto já estava coberto por uma patente húngara, para tratamento da tuberculose, sendo necessária a busca de um composto alternativo; finalmente, um metabólito do $\mathrm{H}$ 124/26 que não estava coberto pela patente húngara se mostrou um potente antissecretor.

Este é um exemplo das dificuldades e estratégias utilizadas durante o desenvolvimento de um novo fármaco. O desenvolvimento passou pela timoprazola, que levava a um aumento da glândula tireoide, além da atrofia do timo, efeito que não era observado em vários mercapto-benzimidazoles. A introdução desses substituintes no timoprazola (Figura 1S, material suplementar) eliminou o efeito na tireoide e no timo, sem que houvesse perda da atividade antissecretora. A continuidade desta linha de trabalho levou à picoprazola (Figura $2 \mathrm{~S}$, material suplementar), que se mostrou ativa em humanos com uma longa duração de ação. O aprimoramento das técnicas de triagem, usando mucosa de cobaias e coelhos, permitiu o aperfeiçoamento dos compostos e a identificação da bomba de próton uma $\mathrm{H}^{+} \mathrm{K}^{+}$-ATPase; a continuidade dessas pesquisas levou à descoberta da omeprazola, como sendo a mais potente testada. ${ }^{11,13,28} \mathrm{Na}$ época do lançamento, já estavam disponíveis medicações para o tratamento de doenças relacionadas ao excesso de acidez gástrica, como os antagonistas seletivos de receptores $\mathrm{H} 2$, classe de fármacos iniciada pela cimetidina (Figura 3S, material suplementar) na década de 1970, sendo responsável por uma revolução do tratamento à época, seguida por outros fármacos da classe (ranitidina, famotidina, nizatidina - Figura $4 \mathrm{~S}$, material suplementar). O surgimento da omeprazola introduz uma nova classe de fármacos, os inibidores específicos irreversíveis das bombas de prótons responsáveis pela secreção ácida das células parietais no estômago.$^{28}$ Esta classe de compostos se mostrou muito eficiente, quando comparada aos fármacos inibidores de $\mathrm{H} 2$, com uma redução da secreção ácida de 80 a 95\%, e um longo período de ação propiciando melhores resultados no tratamento das doenças ácido-pépticas.

Esta nova classe de compostos foi gerada através de diferentes substituições no grupo piridina e/ou na benzimidazola (Figura 2), a molécula gerada é um pró-fármaco, ativado em ambientes ácidos (Figura 7), formando uma sulfenamida tetracíclica gerada por prótons, que tem menor capacidade de atravessar membranas, característica que leva a uma acumulação nas células secretoras ácidas, direcionando a sua ação. Entretanto, o pH ácido estomacal se mostrou como a principal dificuldade, uma vez que o fármaco ativado ainda no trato digestivo tem sua absorção extremamente reduzida. ${ }^{13}$ A conversão na forma de sulfenamida foi também a dificuldade nos diferentes processos de síntese, sendo corrigida pela utilização de $\mathrm{NaOH}$ nas etapas com solvente aquoso, mantendo o ambiente reacional sempre com um $\mathrm{pH}$ acima de $9 .{ }^{17}$

A mesma dificuldade também levou ao desenvolvimento das diferentes formas farmacêuticas, de liberação entérica ou com a adição de compostos alcalinos nas camadas internas dos peletes ou dos comprimidos, permitindo a administração oral da omeprazola. Constituem exemplos de "inovações incrementais" indispensáveis para utilização do novo fármaco desenvolvido.

A década de 1960 teve um marco importante com o surgimento dos casos de teratogênia pela talidomida, despertando novas preocupações nos testes das diferentes moléculas, levando os pesquisadores a investigar a atividade farmacológica e toxicológica dos seus diferentes enantiômeros. ${ }^{29}$ No caso do omeprazola não foi diferente, embora as pesquisas iniciais não tenham mostrado diferenças de perfil farmacocinético entre os enantiomêros em cachorros e em ratos tenha demonstrado maior uma biodisponibilidade da forma (R), o que levou à realização dos primeiros estudos clínicos com o

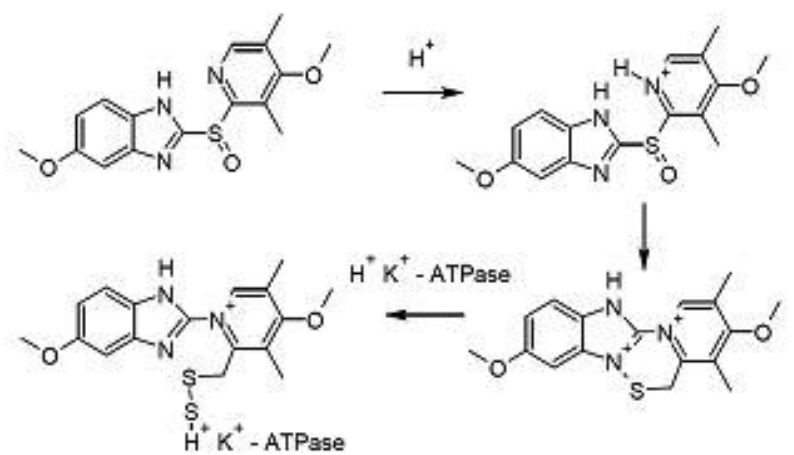

Figura 7. Mecanismo de ativação da omeprazola. A omeprazola é uma base fraca, especificamente concentrada na secreção ácida dos canalículos das células parietais e quando ativada por um próton gera uma sulfenamida. Essa sulfenamida interage covalentemente com os grupos sulfidrilas do resíduo de cisteína no domínio extracelular da $H+K+$-ATPase - Cis 813 - inibindo sua atividade. Adaptada da ref. 4

racemato. ${ }^{28}$ As pesquisas posteriores de farmacocinética em humanos revelaram que o metabolismo das formas $(\mathrm{R})$ e (S) ocorria em velocidades diferentes, levando a um tempo de meia-vida maior para a (S)-omeprazola (esomeprazola). Essa descoberta desencadeou uma série de pesquisas, com o desenvolvimento de diversos processos de resolução do racemato, permitindo a utilização do fármaco enantiomericamente puro..$^{12,30}$

De forma geral, o uso do fármaco enantiomericamente puro apresenta vantagens sobre a mistura racêmica, como aumento da atividade ou redução dos efeitos colaterais, que são realmente são necessárias. Por outro lado, independente da necessidade, essa tem sido uma tendência seguida por diversos laboratórios, mesmo que não haja realmente uma grande vantagem terapêutica. Serve como uma estratégia usada para as patentes mais antigas, próximas de cair em domínio público, ou já em domínio público, permitindo continuar com um produto inovador no mercado e promovendo o relançamento de um fármaco antigo.

Entretanto, o desenvolvimento de enantiômeros exclusivamente com a finalidade de extensão da proteção tende a diminuir, uma vez que o receio do uso dessa estratégia de desenvolvimento pelos concorrentes tem levado à reivindicação das formas enantiomericamente puras logo no primeiro depósito, além da antecipação desse desenvolvimento pelo detentor da primeira patente pouco tempo depois do primeiro depósito. Não obstante, tanto para os casos em que o seu uso se mostre realmente vantajoso, quanto nos casos em que a vantagem não é tão pronunciada, o uso da substância enantiopura tem sido um grande apelo de comercialização junto à classe médica.

Outro aspecto demonstrado no exemplo da omeprazola é o comportamento dos laboratórios concorrentes, sendo que a descoberta de um fármaco para um novo alvo farmacológico desencadeia uma corrida pelo desenvolvimento de moléculas análogas. ${ }^{24,25} \mathrm{~A}$ jornada começa, sem dúvida, muito antes do lançamento pelo concorrente, já que as empresas mantêm monitoramento constante das tecnologias depositadas nos bancos de patente. Na verdade, o primeiro depositante pode desenvolver suas pesquisas sem concorrência nos primeiros 18 meses após o depósito do pedido de patente, período em que o conteúdo depositado permanece em sigilo. Após este período, havendo interesse, outros laboratórios poderão tentar desenvolver tecnologias análogas às depositadas.

O início dos trabalhos é analisar o escopo da proteção, tentando localizar possíveis brechas na fórmula marckush depositada, além de possíveis alterações no processo não protegidas. Desafio que tem ficado cada vez mais difícil, ao passo que as empresas têm se 
especializado em tentar o máximo de proteção no primeiro depósito, procurando descrever todas as moléculas possíveis de serem obtidas através do processo de síntese proposto.

Essas pesquisas podem levar ao descobrimento de novas moléculas com perfil terapêutico melhor - me betters, ou equivalente - me toos. A atividade terapêutica das novas moléculas pode conter melhora no perfil farmacocinético, diferenças no metabolismo, redução de efeitos adversos, aumento na afinidade pelo alvo farmacológico ou mudança na seletividade. ${ }^{4}$

Independente de introduzirem grandes avanços, o surgimento de moléculas análogas fornece opções para o clínico, que pode escolher a molécula da classe que melhor se adapte ao seu paciente por questões terapêuticas, ou mesmo econômicas, uma vez que a quebra de monopólio promovida pelos me toos normalmente leva à redução de preços devido à concorrência.

No caso da omeprazola, tivemos alguns outros medicamentos inseridos no mercado (Figura 4), um número pequeno quando comparado a outras classes terapêuticas e aparentemente sem muita diferença na atividade farmacológica. Entretanto, se olharmos outros exemplos, como os inibidores da enzima conversora de angiotensina (IECA), ${ }^{31}$ ou os antagonistas de receptores beta adrenérgicos (BA), o surgimento de outros fármacos foi não só em número maior, mas introduziu também diferenças importantes em potência (IECA) ou na seletividade (BA). ${ }^{31}$

No caso dos IECA a exploração não só da proteína alvo, mas da via envolvida deu origem a outros fármacos, alguns com reduções significativas dos efeitos adversos, demonstrando que os investimentos em análogos moleculares podem gerar benefícios, tanto para os pacientes, quanto para as empresas que investirem em seu desenvolvimento. ${ }^{5}$

O uso continuado de um fármaco ou a triagem de compostos que chegaram ou não ao mercado para novos alvos farmacológicos pode levar ao desenvolvimento de novos usos terapêuticos do fármaco, sendo outra estratégia utilizada pelos laboratórios de pesquisa no desenvolvimento de novos medicamentos. ${ }^{33}$

Estas estratégias, conhecidas como segundo uso médico, têm sido muito criticadas, principalmente quanto à possibilidade de extensão de patentes, sendo consideradas por alguns países como matéria não privilegiável de proteção patentária, por haver uma interpretação que esse tipo de inovação não atende ao requisito de novidade, previsto em lei, uma vez que se trata de molécula conhecida. Outros países afirmam que a novidade estaria na sua nova aplicação ou no processo de produção de um novo medicamento.

Fugindo da polêmica, anualmente centenas de moléculas são abandonadas em ensaios clínicos nas suas diversas fases de desenvolvimento. Embora essas moléculas não tenham sido eficazes para a finalidade testada, poderiam ser ensaiadas para outra indicação terapêutica, uma vez que o grande número de informações acumuladas nas pesquisas anteriores, incluindo muitas vezes estudos clínicos de fase I, II ou III, podem reduzir os custos de desenvolvimento viabilizando, inclusive, pesquisas de setores tradicionalmente negligenciados pela indústria. ${ }^{34,35}$

Não foi nossa intenção esgotar o assunto, mas demonstrar o impacto que uma nova entidade molecular traz ao mercado e quais os diferentes caminhos que os laboratórios farmacêuticos normalmente adotam na gestão de suas "inovações incrementais ou sequenciais".

Existem críticas de que a indústria farmacêutica utiliza as "inovações incrementais" exclusivamente para a prorrogação da proteção por patentes. Entretanto, os laboratórios farmacêuticos se defendem e argumentam que essas invenções são necessárias e agregam melhorias importantes aos produtos iniciais. ${ }^{4,36,37}$

Sem tomar partido, o exemplo analisado mostrou que algumas “inovações incrementais" são realmente necessárias para viabilização do uso da nova molécula na terapêutica. Além disso, ficou evidente a importância das melhorias de processo, devendo esse tipo de inovação também ser incentivado da mesma forma que as "inovações radicais".

\section{MATERIAL SUPLEMENTAR}

Encontra-se disponibilizado em http://quimicanova.sbq.org.br, na forma de arquivo .PDF, com acesso livre. Apresenta as Figuras $1 \mathrm{~S}$ a 4S.

\section{REFERÊNCIAS}

1. Solano, J. A.; Kirsh, J. M.; Bala, M. V.; Chambers, M. G.; Harpdle, L. H.; Clin.Pharmacol. Ther. 2008, 84, 2.

2. Andersen, M. A.; Innovation, Sustainability and Policy Conference, Kloster Seon: Obing, 2004.

3. Aoki, R.; Kubo, K.; Yamane, H.; WHO-CIPIDH study 2005.

4. Cohen, F. J.; Nat. Rev. Drug Disc. 2005, 4, 78.

5. Pavlou, A. K.; Belsey, M. J.; Nat. Rev. Drug Disc. 2007, 4, 273.

6. Blagden, N.; Matas M.; Gavan, P. T.; York, P.; Adv. Drug Deliv. Rev. 2007, 59, 617.

7. Lima, V. L. E.; Quim. Nova 1997, 20, 657.

8. Ove, G.; Dept. of Industrial Management and Economics Center for Intellectual Property Studies, Chalmer University of Technology, Sweden, 2003.

9. http://www.uspto.gov/patents/process/search/index.jsp, acessada em Março 2010.

10. http://ep.espacenet.com/numberSearch?locale=en_ep, acessada em Março 2010.

11. Berntsson, P. B.; SE pat. 7,402,101 1975.

12. Lovegren, K. L.; US pat. 4,786,505 1988.

13. Brandstrom, A. E.; US pat. 4,738,974 1988.

14. Bengstsson, I. S.; US pat. 5,690,960 1997.

15. Kaellstroem, L. A.; US pat. 5,900,424 1999.

16. Äbelö, A.; Andersson, T. B.; Antonsson, M.; Naudot, A. K.; Skånberg, I.; Weidolf, L.; Drug metab. dispos. 2000, 28, 966.

17. Lindberg, P. L.; US pat. 5,714,504 1998

18. Cotton, H.; US pat. 6,369,085 2002

19. McManus, J. W.; US pat. 6,191,148 2001

20. Bergstrand, P.; US pat. 6,428,810 2002.

21. Chen, C. M.; US pat. 5,260,069 1993.

22. Kamal, K.; US pat. 5,413,777 1995.

23. Lovqvist, K.; US pat. 0,072,913 2007

24. Nohara, A.; US pat. 4,628,098 1986

25. Souda, S.; US pat. 5,045,552 1991

26. Hasselkus, W.; US pat. 5,714,505 1998.

27. Tomoi, M.; Ikeda, Y.; Yokota, Y.; US pat. 5,093,342 1992

28. Barreiro, E. J.; Quim. Nova 2002, 25, 1172.

29. Olbe, C.; Carlsson, E.; Lindberg, P.; Nat. Rev. Drug Disc. 2003, 2, 132.

30. Lima, L. M.; Fraga, C. A. M.; Barreiro, E. J; Quim. Nova 2001, 24, 633.

31. Nick, A.; McManus, J.; US pat. 6,147,103 2000.

32. Acharya, R. K.; Sturrock, E. P.; Riordan, J. F.; Ehlers, M. R.; Nat. Rev. Drug Disc.2003, 2, 891.

33. Kaye, D.; Krum, H.; Nat. Rev. Drug Disc. 2007, 6, 127.

34. Chong, C. R.; Sullivan, D. J.; Nature 2007, 448, 645.

35. Moorman, A. E.; Becker, D. P.; Flynn, D. L.; Li, H.; Villamil, C. I.; US pat. 5,945,425 1999

36. Darrow, J.; Stan. Tech. L. Rev. 2007; disponível em http://stlr.stanford. edu/pdf/darrow_patentability.pdf.

37. Krankii, R. K.; Grant, D. J. W; Thermochim. Acta 1995, 248, 61. 


\section{GESTÃO DAS INOVAÇÕES INCREMENTAIS, O CASO OMEPRAZOLA}

\section{Alexandre Lopes Lourenço* e Luiz Marcelo Lira}

Instituto Nacional da Propriedade Industrial, Rua Mayrink Veiga, 9, 20090-910 Rio de Janeiro - RJ, Brasil

Denise Pires de Carvalho

Instituto de Biofísica Carlos Chagas Filho, Centro de Ciências da Saúde, Bl. G, Universidade Federal do Rio de Janeiro, Ilha do Fundão, 21949-900 Rio de Janeiro - RJ, Brasil

Paulo de Assis Melo e Tereza Sollero Cláudio-da-Silva

Instituto de Ciências Biomédicas, Centro de Ciências da Saúde, Bl. K, Universidade Federal do Rio de Janeiro, Ilha do Fundão, 21941-590 Rio de Janeiro - RJ, Brasil

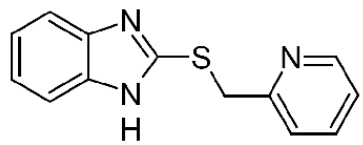

Figura 1S. Timoprazola<smiles>COC(=O)c1cc2[nH]c(SCc3ncccc3C)nc2cc1C</smiles>

Figura 2S. Picoprazola<smiles>CN=C(NC)NCCSCc1nc[nH]c1C</smiles>

Figura 3S. Cimetidina

*e-mail: allopes@inpi.gov.br<smiles>CN(C)Cc1ccc(CSCCN/C(=C\[N+](=O)[O-])N(C)C)o1</smiles>

A<smiles>CN/C(=C\[N+](=O)[O-])NCCSCc1csc(N=C(C)C)n1</smiles>

B<smiles>CN(C)Cc1nc(CSCCN/C(=C/[O-])N(C)C)cs1</smiles>

Figura 4S. Análogos moleculares da cimetidina: ranitidina (A), famotidina (B) e nizatidina $(C)$ 